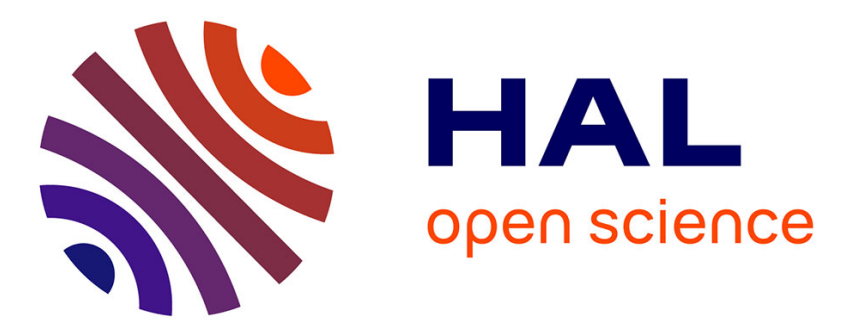

\title{
Intercalation and ordering of F 6 TCNNQ and F 4 TCNQ dopants in regioregular poly(3-hexylthiophene) crystals: impact on anisotropic thermoelectric properties of oriented thin films
}

Viktoriia Untilova, Huiyan Zeng, Pablo Durand, Laurent Herrmann, Nicolas Leclerc, Martin Brinkmann

\section{To cite this version:}

Viktoriia Untilova, Huiyan Zeng, Pablo Durand, Laurent Herrmann, Nicolas Leclerc, et al.. Intercalation and ordering of F 6 TCNNQ and F 4 TCNQ dopants in regioregular poly(3-hexylthiophene) crystals: impact on anisotropic thermoelectric properties of oriented thin films. Macromolecules, 2021, 10.1021/acs.macromol.1c00554 . hal-03405478

\author{
HAL Id: hal-03405478 \\ https://cnrs.hal.science/hal-03405478
}

Submitted on 27 Oct 2021

HAL is a multi-disciplinary open access archive for the deposit and dissemination of scientific research documents, whether they are published or not. The documents may come from teaching and research institutions in France or abroad, or from public or private research centers.
L'archive ouverte pluridisciplinaire HAL, est destinée au dépôt et à la diffusion de documents scientifiques de niveau recherche, publiés ou non, émanant des établissements d'enseignement et de recherche français ou étrangers, des laboratoires publics ou privés. 
Intercalation and ordering of $\mathrm{F}_{6}$ TCNNQ and $\mathrm{F}_{4}$ TCNQ dopants in regioregular poly(3-hexylthiophene) crystals: impact on anisotropic thermoelectric properties of oriented thin films

Viktoriia Untilova, ${ }^{1}$ Huiyan Zeng ${ }^{1}$, Pablo Durand ${ }^{2}$, Laurent Herrmann ${ }^{1}$, Nicolas Leclerc ${ }^{2}$ and Martin Brinkmann ${ }^{1 *}$

(1) Université de Strasbourg, CNRS, ICS UPR 22, F-67000 Strasbourg, France

(2) Université de Strasbourg, CNRS, ICPEES UMR 7515, F-67087 Strasbourg, France

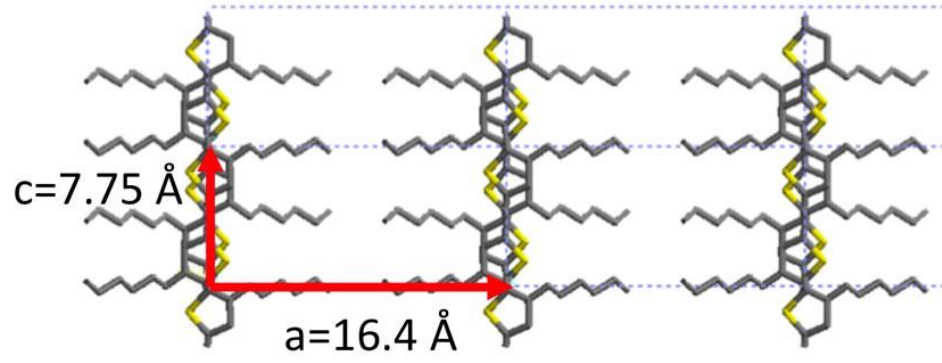

Doping $\square F_{6}$ TCNNQ

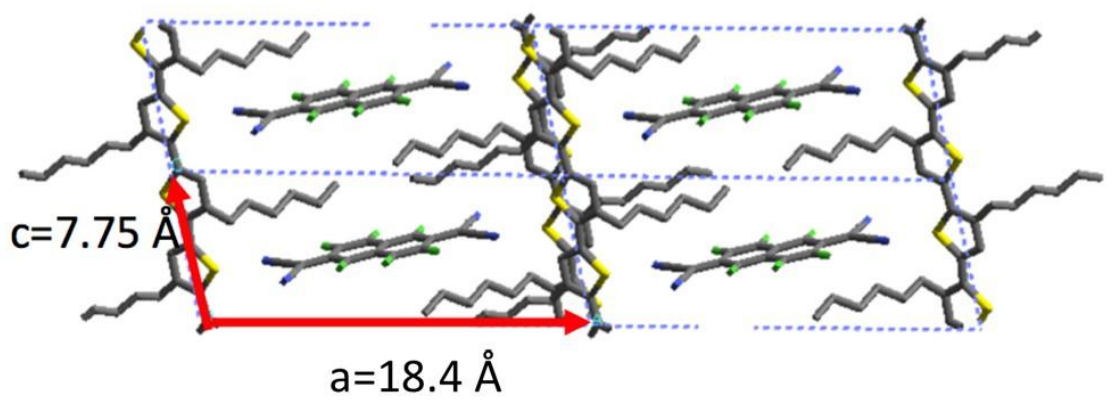




\begin{abstract}
Redox doping is widely used to transform polymer semiconductors such as regioregular poly(3-hexylthiophene) into conducting polymers. Strong acceptor molecules such as $\mathrm{F}_{6}$ TCNNQ and $\mathrm{F}_{4}$ TCNQ have been used for sequential doping of aligned P3HT films to produce films with enhanced thermoelectric properties along the chain direction. This study uses Polarized UV-vis-NIR spectroscopy and electron diffraction to clarify the way dopant molecules are introduced in the crystals of P3HT. A structural model is obtained for F6 TCNNQ-doped P3HT (P-1 symmetry, $a=18.8 \AA, b=8.95 \AA, c=7.75 \AA, \alpha=107.6^{\circ}, \beta=101.5^{\circ}$ and $\gamma=89.3^{\circ}$ ). The proposed structure is consistent with the electron diffraction pattern and polarized UV-vis-NIR results. The model predicts intercalation of one dopant per four thiophene monomers, reduced $\pi$-stacking distance and expanded unit cell along alkyl side chains. Both TEM and polarized absorption spectroscopy demonstrate that $\mathrm{F}_{6} \mathrm{TCNNQ}$ molecules are better ordered than $\mathrm{F}_{4} \mathrm{TCNQ}$ in the P3HT crystals, leading to improved charge conductivities reaching $500 \mathrm{~S} / \mathrm{cm}$ with thermoelectric power factors close to $80 \mu \mathrm{W} \mathrm{m} \mathrm{K}^{-1}$.
\end{abstract}

* Corresponding authors.

martin.brinkmann@ics-cnrs.unistra.fr 


\section{Introduction.}

In the last decades, polymer semiconductors have gained considerable attention since they combine remarkable optical and electronic properties with mechanical flexibility, light weight and low-temperature processing. [1] Numerous fields of applications exist, to cite but a few: organic photovoltaics, organic field effect transistors and, more recently, the design of thermoelectric (TE) generators. From a material's perspective, TE properties are observed in polymer semiconductors upon doping and can be adjusted by numerous methods e.g. by tailoring the polymer backbone, tuning the length of side chains, adjusting the energetic offset between the polymer's HOMO and the acceptor's LUMO, tuning the chemical structure of the dopant or choosing the best doping method. [2-5] Much attention is currently focused on doped p-type polymer semiconductors (PSCs) suitable for thermoelectric applications. Polythiophenes such as regioregular poly(3-hexylthiophene) (P3HT), poly(3,4ethylenedioxythiophene)-tosylate (PEDOT-Tos) or Poly[2,5-bis(3-alkylthiophen-2yl)thieno[3,2-b]thiophene] (PBTTTs) doped with acceptor molecules e.g. 2,3,5,6-Tetrafluoro7,7,8,8-tetracyanoquinodimethane ( $\left.\mathrm{F}_{4} \mathrm{TCNQ}\right)$ or $\mathrm{FeCl}_{3}$ have been investigated intensively. [612] $\mathrm{F}_{4}$ TCNQ-doped P3HT emerged as a model system to better understand the correlations between processing, structure and TE properties [12-15].

Doping of PSCs is the important step that transforms the pristine semiconducting polymer into conducting ones. Doping controls the conductivity of the films and also the resulting TE power factors $(\mathrm{PF})$ that scales with the square root of the charge conductivity $\sigma$. [16] Redox doping rests on a charge transfer between the polymer and the dopant. Integer charge transfer (ICT) occurs from the HOMO $\sim-4.8 \mathrm{eV}$ of P3HT to the LUMO $\sim-5.2 \mathrm{eV}$ of $\mathrm{F}_{4}$ TCNQ. [17] The position of the LUMO of the acceptor is essential and must be well adapted to the HOMO of the polymer. Of particular interest are acceptor molecules with lower LUMO than F 4 TCNQ. For example, 1,3,4,5,7,8-Hexafluorotetracyanonaphthoquinodimethane 
( $\mathrm{F}_{6} \mathrm{TCNNQ}$ ) exhibits higher electronic affinity $\left(-5.37 \mathrm{eV}\right.$ ) than $\mathrm{F}_{4} \mathrm{TCNQ}(-5.2 \mathrm{eV})$ (see Figure 1a) and has been used successfully to dope P3HT and low band gap polymers. $[18,19]$

Beside the chemical nature of the dopant, the doping method is also a key factor. Various doping methods have been investigated such as mixed solution/sequential processing [5,13,16], vapor phase doping [20], electrochemical doping [21,22] and more recently incremental concentration doping (ICD). [23] Mixed polymer/dopant solution processing $[24,25]$ is quite ineffective due to the poor resulting film quality and low TE performances. In strong contrast, with sequential doping, the dopant molecules are intercalated into the crystals of PSCs and that the pristine order of the polymer is preserved upon doping. [13,14] Vaporphase doping is also very effective as it allows to intercalate dopant molecules progressively into the pristine polymer structure without the need for an organic solvent that can interact with the polymer during doping. [12] Similar to vapor phase doping, a variant of sequential doping can lead to improved TE properties in $\mathrm{F}_{6}$ TCNNQ-doped PBTTT (see Figure 1.b). In this Incremental Concentration Doping (ICD) technique, increasing amounts of dopants are progressively introduced into the PSC films. It allows to reach higher conductivities in comparison to direct doping due to the gradual dopant intercalation e.g. for PBTTT films doped by F $_{6}$ TCNNQ. [23]

The dopant location also impacts the final TE properties of semi-crystalline PSCs whether it enters in the crystalline part only or in both crystalline and amorphous domains. There is a general consensus that the observed result depends on the dopant, the doping method and the solvent used for doping. [5,12,17-20] Several recent studies on P3HT and PBTTT have demonstrated that the dopant molecules are located in the layers of alkyl side chains whereas the amorphous part remains little-doped with $\mathrm{F}_{4} \mathrm{TCNQ}$ [3, 26, 27]. Limited doping of amorphous P3HT is possibly due to the different energetic offsets between the dopant's LUMO and the different HOMO levels in the crystalline and amorphous zones. Several tenth 
of $\mathrm{eV}$ difference between crystalline and amorphous zones can already complicate the charge transfer between polymer and dopant. [28] It has been recently demonstrated that bulkier dopant molecules such as Molybdenum complex (e.g. $\left.\mathrm{Mo}\left(\mathrm{tfd}-\mathrm{COCF}_{3}\right)_{3}\right)$, exhibiting higher electron affinities $(\sim-5.6 \mathrm{eV})$, are able to dope both amorphous and crystalline regions of P3HT. $[29,30]$

The polymer's crystallinity in the pristine state prior to doping is equally important for TE properties. Hynynen et al. established the correlation between the initial crystallinity of P3HT and the final conductivity after doping with $\mathrm{F}_{4}$ TCNQ.[18] Scholes et al. showed that a higher film crystallinity leads to higher mobility and greater polaron delocalization. [12] Similarly, in oriented films of P3HT, both improved alignment and crystallinity have improved TE properties. [26,27] Exploiting the anisotropic transport of PSCs is a further means to improve TE properties of doped PSCs because charge conductivity is usually maximal along the chain and $\pi$-stacking directions. [26,27,30] In this regard, high-temperature rubbing proved highly effective because both alignment and film crystallinity can be readily controlled by adjusting the temperature of rubbing $T_{R}$. [31] On average, a ten-fold increase in conductivity is observed parallel to the chain direction in doped and oriented P3HT and PBTTT films as compared to isotropic thin films $[17,26,27,30]$. More interestingly, polymer alignment also enhances the Seebeck coefficient and is therefore a simple method to reach larger power factors in thin films.

In this contribution we investigate the effect of the dopant on optical, structural and thermoelectric properties in aligned P3HT films doped sequentially with $\mathrm{F}_{4} \mathrm{TCNQ}$ and F $_{6}$ TCNNQ. We evaluate how the dopant size and electron affinity affect the final film structure as a function of dopant concentration. We use a combination of polarized UV-VisNIR spectroscopy and low dose electron diffraction to evaluate how the doping by different molecules affects the final polymer structure and how the dopant molecules are distributed in 
the P3HT crystals. Measurements of DC conductivity and Seebeck coefficients in anisotropic films help to correlate doped polymer structure and TE properties. Differences in doping mechanism and film structure versus dopant concentration are evidenced. Both absorption spectroscopy and electron diffraction provide compelling evidence for the intercalation of $\mathrm{F}_{6}$ TCNNQ dopants inside the crystal lattice of P3HT. A tentative model for the structure of $\mathrm{F}_{6}$ TCNNQ-doped P3HT is obtained from ED analysis.
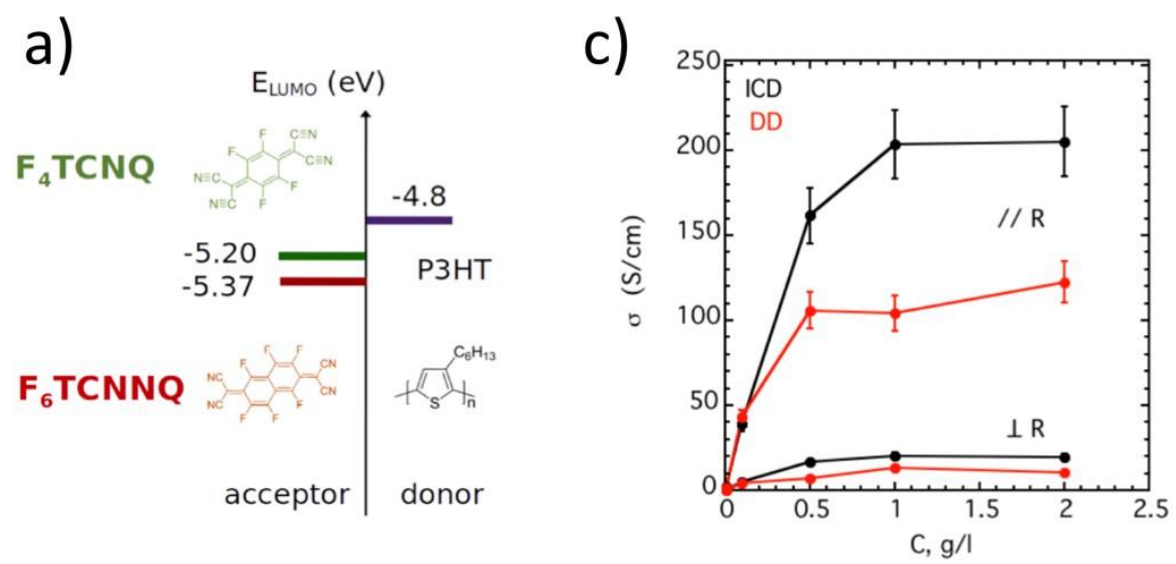

b)

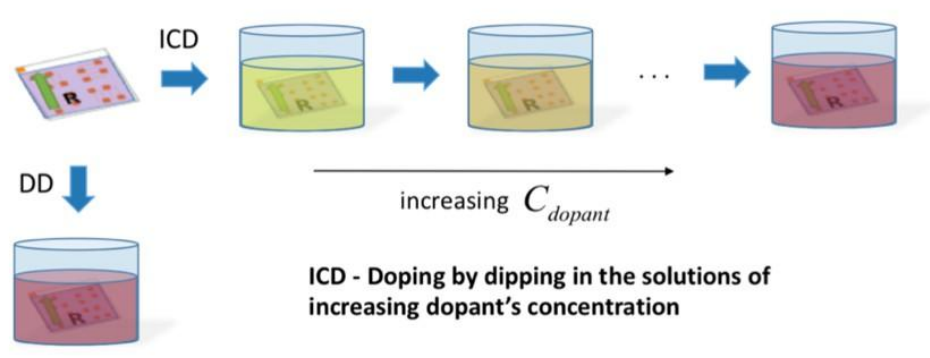

Figure 1. a) Energy diagram of the LUMO levels of $F_{4}$ TCNQ, $F_{6}$ TCNNQ and HOMO of P3HT, b) Schematic illustration of incremental concentration doping (ICD) and direct doping (DD) methods, c) Comparison of the electrical conductivity of oriented P3HT films doped with $\mathrm{F}_{4} \mathrm{TCNQ}$ in acetonitrile by the two doping methods as a function of increasing dopant concentration.

\section{Results}




\subsection{Incremental Concentration Doping (ICD) versus Direct Doping (DD).}

Firstly, we verify if the ICD protocol leads to higher charge conductivities as compared to the direct doping (DD) method used for sequential doping. We therefore compare the charge conductivities in thin films doped by ICD and DD with $\mathrm{F}_{4}$ TCNQ in acetonitrile. In ICD, for a given doping concentration $C_{\text {dopant }}$, the sample has been doped at low but increasingly higher doping concentrations (see Figure 1b). As seen in Figure 1c, ICD allows to reach larger charge conductivities using the ICD method. The conductivity at $1 \mathrm{~g} / \mathrm{l}$ is improved by a factor 1.6 using ICD instead of DD, reaching a conductivity parallel to the rubbing of $205 \mathrm{~S} / \mathrm{cm}$ for $\mathrm{F}_{4} \mathrm{TCNQ}$ versus $122 \mathrm{~S} / \mathrm{cm}$ for DD. The improvement is comparable to that observed when ICD was applied to $\mathrm{C}_{12}$-PBTTT. [23] ICD appears to be a valid option for other polymer semiconductors. In the case of $\mathrm{C}_{12}$ PBTTT, we have shown that ICD and DD lead to similar apparent charge carrier densities since the polaronic absorption bands are nearly identical. Therefore, it was concluded that ICD leads to a higher charge mobility because the structure of the polymer is better preserved when the dopants are introduced in a progressive manner into the polymer crystals. Likewise, we used ED to check whether ICD and DD correspond to different structural characteristics of the doped films. As an example, Figure ESI1 depicts diffraction patterns of $\mathrm{F}_{4}$ TCNQ-doped P3HT at 2g/l by ICD and by DD. No significant structural changes can be evidenced by electron diffraction apart from a marginal change in the $d_{100}$. Similarly, for $F_{6} T C N N Q$, no substantial structural changes are visible between DD and ICD. Differences in microstructure between the two types of doped films using the two methods are accordingly quite subtle and cannot be evidenced by ED.

\subsection{Spectroscopic signatures of doping.}

The difference in the doping mechanism of oriented P3HT with $\mathrm{F}_{4}$ TCNQ and $\mathrm{F}_{6}$ TCNNQ was first probed using polarized UV-vis-NIR absorption spectroscopy. Figure 2 a,b shows the 
polarized UV-vis spectra of aligned P3HT films doped by $\mathrm{F}_{4}$ TCNQ and by $\mathrm{F}_{6} \mathrm{TCNNQ}$ at $5 \mathrm{~g} / \mathrm{l}$. Both polaronic and anionic signals are strongly polarized with respect to the rubbing direction. As seen in Figure 2a, the $\mathrm{F}_{4} \mathrm{TCNQ}$ anion has characteristic absorption peaks at 413, 690, 770 and $873 \mathrm{~nm}$ polarized perpendicular to the rubbing direction. [26,30] In the direction of rubbing, the spectrum is dominated by the neutral polymer fraction at $525 \mathrm{~nm}$ and the two polaronic P1 and P2 bands located respectively at $2500 \mathrm{~nm}$ and $795 \mathrm{~nm}$. As for F $_{6}$ TCNNQ (Figure 2b) the main anionic absorption peaks are centered at 864, 988 and 1143 $\mathrm{nm}$ and polarized perpendicular to the rubbing. A few additional $\mathrm{F}_{6} \mathrm{TCNNQ}^{-}$absorption peaks are centered at 414, 474 and $502 \mathrm{~nm}$. The polaronic absorption peaks P1 and P2 are located at the same positions as in case of $\mathrm{F}_{4} \mathrm{TCNQ}$ and are polarized along the rubbing direction. The relative polarizations of the dopant anions and the polaron indicates that both $\mathrm{F}_{4} \mathrm{TCNQ}$ and $\mathrm{F}_{6}$ TCNNQ are oriented with their long axis in a plane perpendicular to the P3HT chain direction. For POL//R, a higher ratio between the $\mathrm{P} 1$ and the neutral $\mathrm{N}$ polymer absorption bands is observed for the films doped with $\mathrm{F}_{6}$ TCNNQ. This is consistent with a higher oxidation level of P3HT by $\mathrm{F}_{6} \mathrm{TCNNQ}$ as compared to $\mathrm{F}_{4} \mathrm{TCNQ}$, as expected from the lower LUMO level of $\mathrm{F}_{6} \mathrm{TCNNQ}$ versus $\mathrm{F}_{4} \mathrm{TCNQ}$.

Following the absorbance of the polaronic P1 band versus doping concentration helps evidence further differences in the doping process. At very low concentration (below $10^{-4}$ $\mathrm{Mol} / \mathrm{l}$ ), there is already a strong P1 band for $\mathrm{F}_{4} \mathrm{TCNQ}$ whereas for $\mathrm{F}_{6} \mathrm{TCNNQ}$ the absorbance is substantially lower. Only for $\mathrm{C} \geq 3 \cdot 10^{-3} \mathrm{Mol} / \mathrm{l}$ are similar P1 absorbances observed for both dopants (see Figure ESI 2). The doping level (dopant per thiophene) can be estimated from the absorbance of the anions, knowing the extinction coefficients $\varepsilon$ for both dopants and the film thickness (see experimental section). As explained in our previous work, the determination of the doping level from the anion absorbance is not valid if most of the dopant molecules lie with their long axis in the film plane as the transition dipole is parallel to the molecular long axis of the dopant molecules. [27] This is indeed the case for rubbed films 
that are made essentially of face-on P3HT crystals. As seen in Figure 2d, the final apparent doping level is larger for $\mathrm{F}_{6} \mathrm{TCNNQ}\left(20 \pm 2\right.$ dopants/100 thiophene) compared to $\mathrm{F}_{4} \mathrm{TCNQ}$ (14 \pm 2 dopants per 100 thiophenes). The larger doping with F $_{6}$ TCNNQ is consistent with the lower LUMO level of $\mathrm{F}_{6} \mathrm{TCNNQ}$ relative to $\mathrm{F}_{4} \mathrm{TCNQ}$. Additionally, we confirm the validity of ICT mechanism for both dopants (see Figure ESI 3).

a)

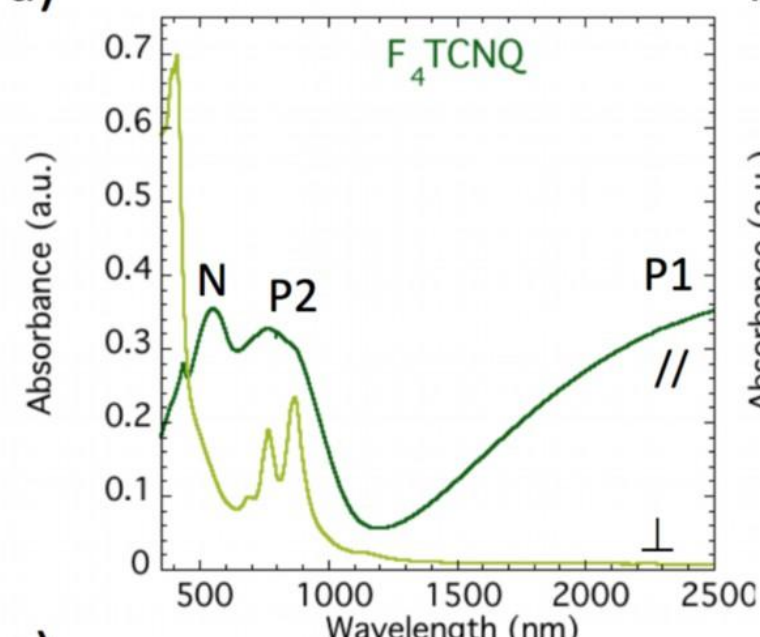

c)

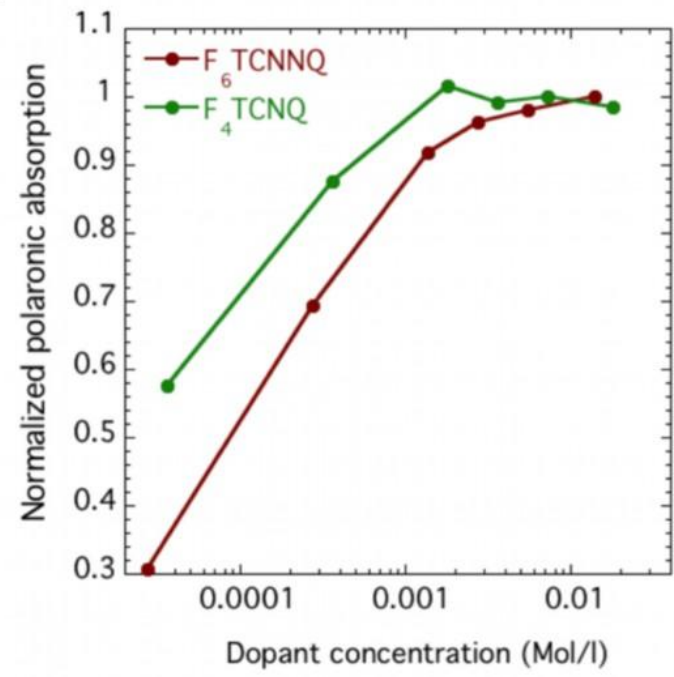

b)

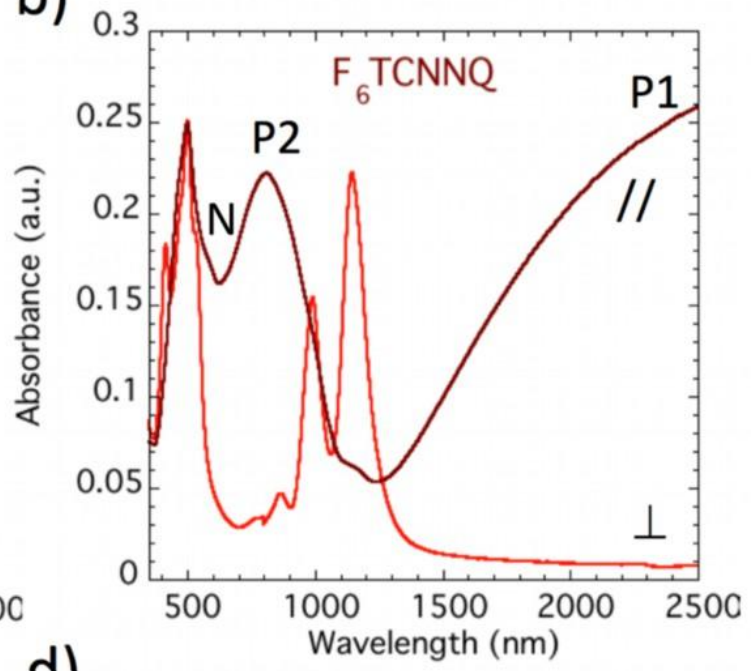

d)

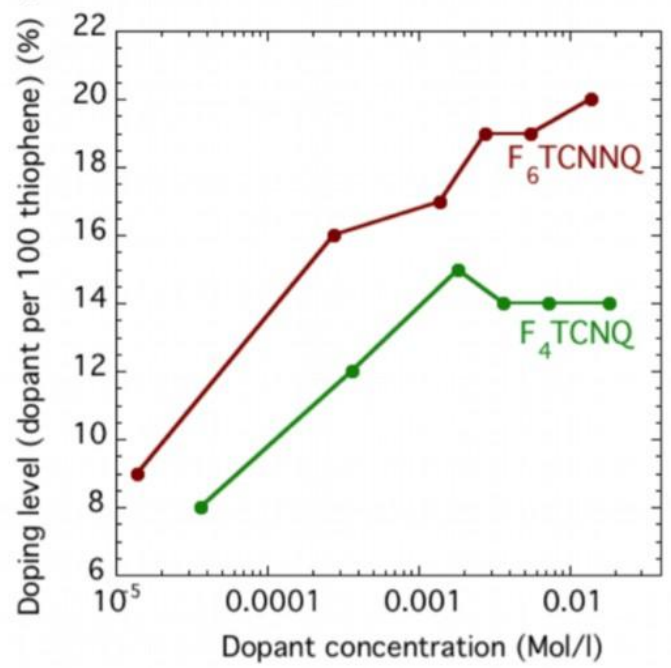

Figure 2. Polarized UV-vis-NIR spectra of oriented P3HT films featuring highly polarized polaronic absorption bands $(P 1, P 2)$ for incident light polarized parallel to the rubbing direction (11) and the polarized absorption bands of the anion for incident light polarized 
perpendicular to the rubbing direction ( $\perp$ ). P3HT films are sequentially doped using a $5 \mathrm{~g} / \mathrm{l}$ solution in acetonitrile of a) F4TCNQ and b) $F_{6} T C N N Q$. c) Polaronic absorption at $2500 \mathrm{~nm}$ as a function of the dopant concentration. Normalization is done with respect to the maximum absorbance of P1 for each dopant. d) Doping levels obtained from the anion absorbance versus dopant concentration during the ICD protocol (see text for the calculation method). Green and dark red curves correspond to $F_{4} T C N Q$ and $F_{6} T C N N Q$, respectively.

\subsection{Structural differences between $\mathrm{F}_{4}$ TCNQ- and $\mathrm{F}_{6}$ TCNQ-doped P3HT films}

a) Evolution of the structure with doping concentration.

Figure 3 compares the structural modifications followed by electron diffraction (ED) in aligned P3HT films. It shows the ED patterns of oriented P3HT doped sequentially with 0.01 $\mathrm{g} / \mathrm{l}$ and $2.0 \mathrm{~g} / \mathrm{l}$ solutions of $\mathrm{F}_{4} \mathrm{TCNQ}$ and $\mathrm{F}_{6} \mathrm{TCNNQ}$ in acetonitrile. The section profiles along the equatorial reflections from the complete set of experiments at different doping concentrations using incremental concentration are depicted in figures $3 \mathrm{c}$ and 3.f.

For $\mathrm{F}_{4} \mathrm{TCNQ}$, the structure of oriented P3HT films shows an expansion of the unit cell along the alkyl side chain direction from $16.4 \AA$ up to $18.1 \AA$ and the $\pi$-stacking distance is reduced from $3.8 \AA$ to $3.6 \AA$ already for a doping concentration as low as $0.01 \mathrm{~g} / \mathrm{l}$. (Figure $4 \mathrm{a}$ ) As seen in Figure 3c, once the doped phase is formed at $0.01 \mathrm{~g} / 1$, structural changes are marginal upon doping at higher concentrations. This is consistent with the absorption data showing that the polaron intensity changes only slightly above $0.1 \mathrm{~g} / \mathrm{l}$. As observed in our previous study, the $\mathrm{h}$ 02 reflections present in the pristine oriented P3HT films [26,27] are replaced by an intense and streaked 002 reflection. This is a clear indication for the intercalation of $\mathrm{F}_{4} \mathrm{TCNQ}$ in the P3HT crystals that leads to the shifts between planes of $\pi$-stacked P3HT chains to accommodate the dopants in the lattice. [27] 


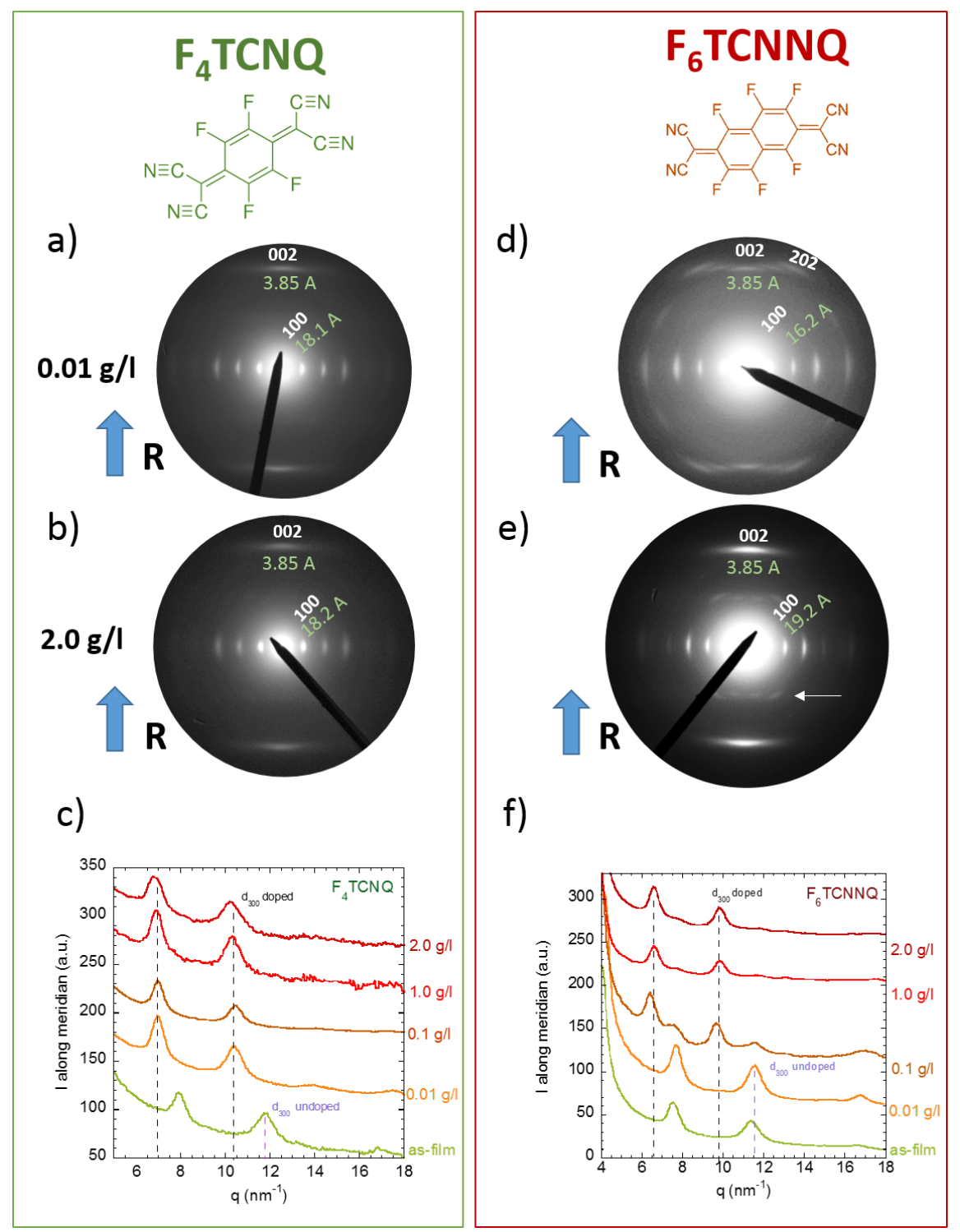

Figure 3: Electron diffraction patterns of oriented P3HT films doped with $F_{4} T C N Q(a, b)$ and with $F_{6} T C N N Q(d, e)$ for doping concentrations of $0.01 \mathrm{~g} / \mathrm{l}(a, d)$ and $2.0 \mathrm{~g} / \mathrm{l}(\mathrm{b}, \mathrm{e})$, respectively. c) and f): equatorial section profiles of the electron diffraction patterns at different doping concentrations for $F_{4} T C N Q(c)$ and for $F_{6} T C N N Q(f)$. For clarity, the section profiles are shifted along the ordinate axis and the intensity of the patterns are normalized to the intensity of the 200 peak. The rubbing direction $\mathbf{R}$ (blue arrow) is vertical for all patterns. The 002 reflection was used to calibrate the ED patterns. In the diffraction pattern (e), additional h 01 reflections related to the $F_{6} T C N N Q$-doped P3HT phase are indicated by a white arrow. 
The situation in the case of $\mathrm{F}_{6} \mathrm{TCNNQ}$ doping is different (see ED patterns and corresponding section profile along the equator in Figure $3 \mathrm{~d}$, e, f). The diffraction pattern for $0.01 \mathrm{~g} / \mathrm{l}$ is identical to the undoped phase, indicating apparently no intercalation of $\mathrm{F}_{6}$ TCNNQ at this concentration although some polaronic and anion bands are already visible for this concentration. For $0.1 \mathrm{~g} / \mathrm{l}$ the doped and undoped phases coexist as indicated by the presence of two 100 peaks with different $d_{100}$ periodicities corresponding to the $F_{6}$ TCNNQ-doped P3HT phase (19.2 $\AA$ ) and to the undoped P3HT phase (16.4 $\AA$ ) (see also Figure 4b). As for $\mathrm{F}_{4} \mathrm{TCNQ}$, the intercalation of dopants results in changes of the ED patterns in terms of reflection intensity. The h $02(\mathrm{~h}=1,2)$ reflections disappear and are replaced by a streaked and very intense 002 . Interestingly, for $2.0 \mathrm{~g} / \mathrm{l}$, the ED pattern is typical of the pure doped phase without any trace of undoped P3HT. Accordingly, doping is more progressive in the case of $\mathrm{F}_{6}$ TCNNQ, which means that doped and undoped phases with $\mathrm{F}_{6}$ TCNNQ may coexist. This observation may indicate that dopant diffusion inside the alkyl side chain layers is more difficult for the larger $\mathrm{F}_{6} \mathrm{TCNNQ}$. Coexistence of doped and undoped phases may also point at a spatially inhomogeneous distribution of dopants in the films for the concentrations below $0.1 \mathrm{~g} / \mathrm{l}$. ED analysis shows that the $\mathrm{F}_{6} \mathrm{TCNNQ}$-doped phase has additional h 01 reflections (Figure 3.e). Since these new reflections are absent in the pristine P3HT films, the doping has substantially modified the original structure of P3HT. The enhanced intensity of the 002 is synonymous of shifted/translated PT backbones within $\pi$ stacks. [27] Overall, the final $d_{100}$ periodicity is larger for $F_{6}$ TCNNQ (19.1 $\AA$ ) than for $\mathrm{F}_{4}$ TCNQ $(18.2 \AA)$, which is consistent with the larger long axis dimension for $\mathrm{F}_{6} \mathrm{TCNNQ}$ whereas the $\pi$-stacking periods are quite similar in both doped phases. A detailed structural analysis is presented in the following for $\mathrm{F}_{6}$ TCNNQ-P3HT. 

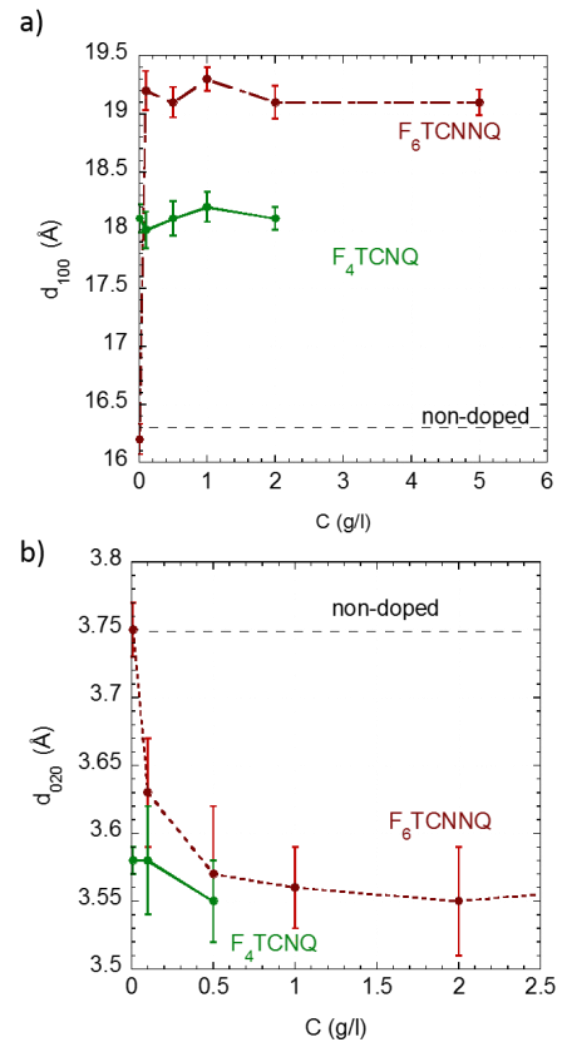

Figure 4: Evolution of the layer spacing along the alkyl side chain direction d100 (a) and the $\pi$-stacking periodicity (b) as a function of doping concentration. The curves in green (dark red) correspond to the F4TCNQ $\left(F_{6} T C N N Q\right)$ doped films.

\section{b) Structural model for P3HT: F $_{6}$ TCNNQ.}

For a $\mathrm{F}_{6} \mathrm{TCNNQ}$ concentration of $5 \mathrm{~g} / \mathrm{l}$, ED indicates that the film has undergone a substantial structural re-organization. The ED patterns of pristine (form I) and $\mathrm{F}_{6} \mathrm{TCNNQ}$-doped phases of $\mathrm{P} 3 \mathrm{HT}$ are illustrated and compared in Figure 5. Beside the changes in the lattice periodicity along the alkyl side chains (a axis) and the $\pi$-stacking direction (b axis), the ED pattern of the doped phase shows additional reflections in the $\ell=1$ layer line. In the pristine form I of P3HT, all $h k 1$ reflections are forbidden for symmetry reasons ( $2_{1}$ axis along c). Close inspection of the ED pattern indicates that the additional reflections cannot be indexed as $h 01$ since they are not located on the $\mathrm{h}=1$ and $\mathrm{h}=2$ layer lines (see Figure ESI 4 ) but are situated on intermediate layer lines. The new reflections can be indexed assuming that the unit cell of the doped phase is doubled along the a axis (side chain direction). 

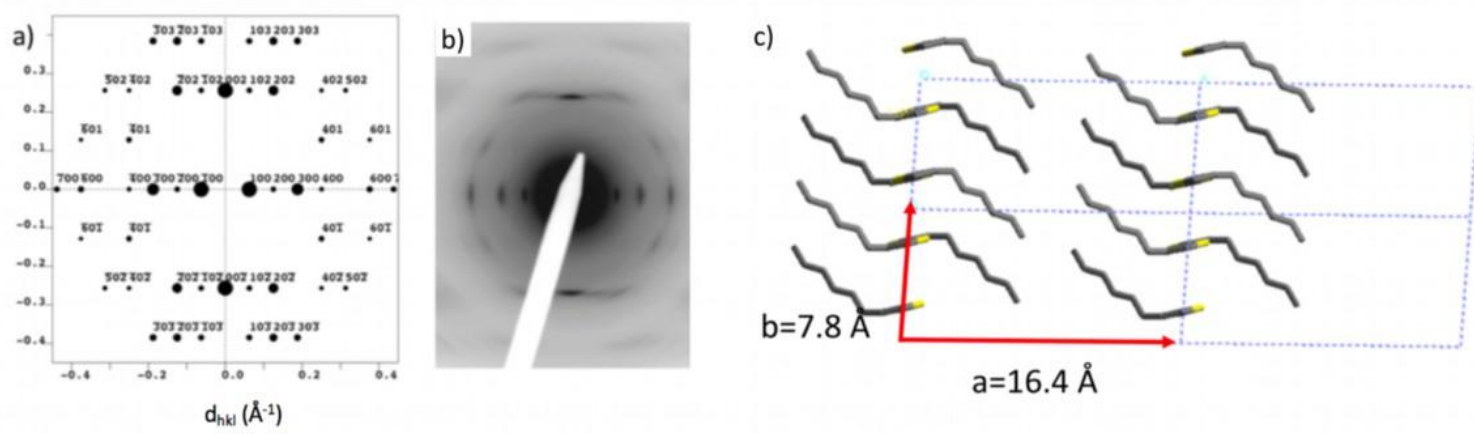

$a=16.4 \AA$
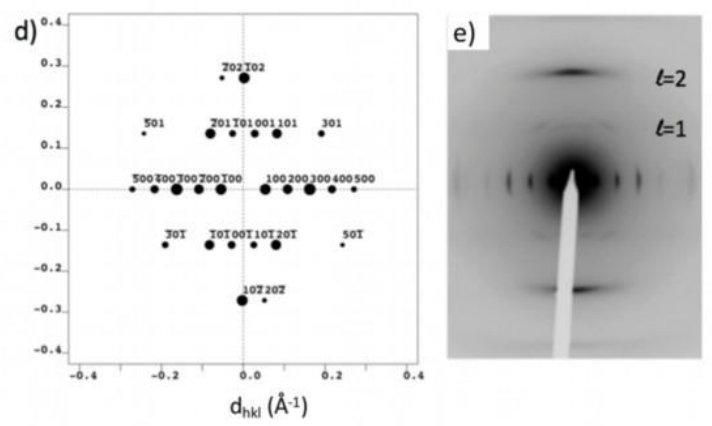

f)

(3 $\left.\begin{array}{lll}3 & 0\end{array}\right)$

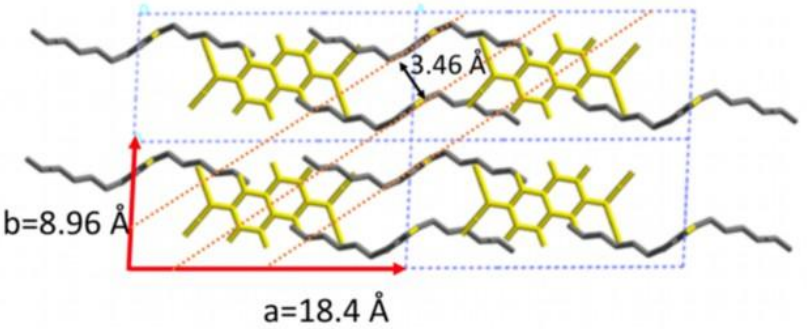

Figure 5. Calculated $(a$ and $d$ ) and experimental ( $b$ and e) electron diffraction patterns for the [l $\left.\begin{array}{lll}0 & 1 & 0\end{array}\right]$ zone for P3HT (form I) ( $a$ and $b$ ) and P3HT-F TCNNQ ( $d$ and $e$ ). The form I structure is taken from reference [30] whereas the doped P3HT structure is refined and corresponds to the model illustrated in Figure 6. In c) and f), the c-axis projection of the pristine and doped structures are shown, respectively.

Both, polarized UV-vis-NIR and electron diffraction provide evidence for the inclusion of dopant molecules in the crystal lattice of P3HT. This intercalation provides an ordered arrangement of dopants in the P3HT lattice, albeit with some statistical disorder. Therefore, to establish a structural model of $\mathrm{P} 3 \mathrm{HT} / \mathrm{F}_{6} \mathrm{TCNNQ}$, it is essential to understand the intercalation mechanism of the dopant molecules in the P3HT crystals.

Let us start with the structure of undoped P3HT (form I) as depicted in Figure 5. In the following, we use the structural model by Kayunkid et al. that was established by electron diffraction analysis. [32] In form I, a dense packing of side chains is observed thanks to the $\Delta \mathrm{c}$ shift between two successive $\pi$-stacked P3HT backbones (Figure 6.a). Therefore, there are no "cavities" to host dopant molecules in pristine P3HT. To intercalate $\mathrm{F}_{4}$ TCNQ or $\mathrm{F}_{6} \mathrm{TCNNQ}$ in the P3HT unit cell, it is necessary to modify the $\pi$-stacking of P3HT chains within the individual $\pi$-stacks $((\mathrm{b}, \mathrm{c})$ planes). As noted in our previous study for 
$\mathrm{P} 3 \mathrm{HT} / \mathrm{F}_{4} \mathrm{TCNQ}$, ED indicates that the doping mechanism induces a structural reorganization of polymer chains within $(b, c)$ planes in the P3HT crystal. [27]

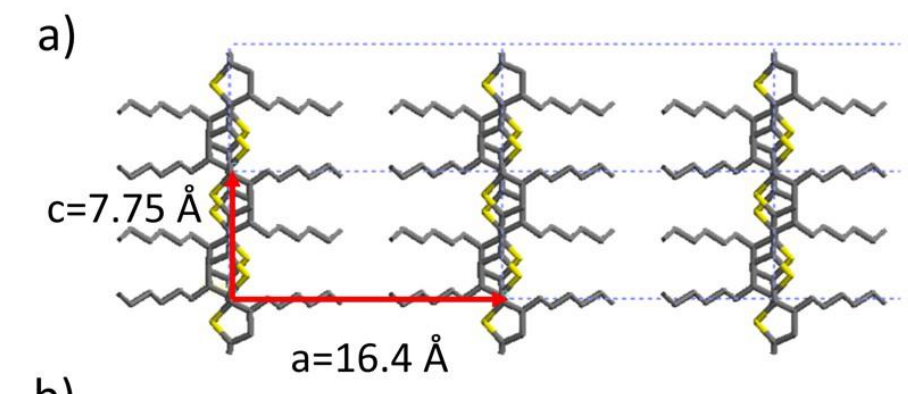

b)

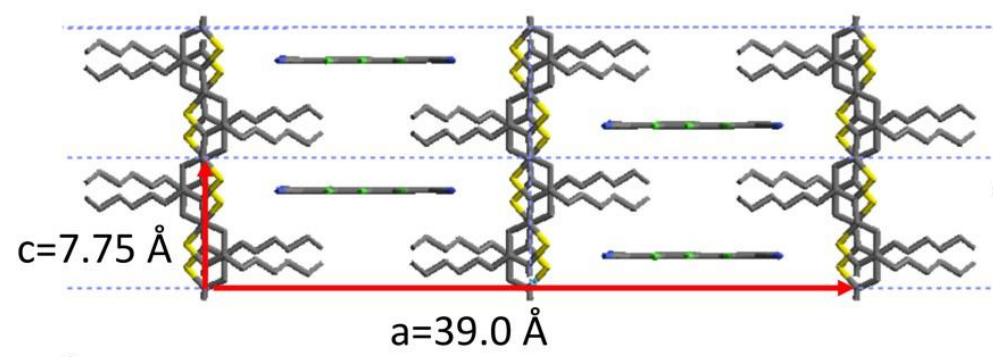

c)

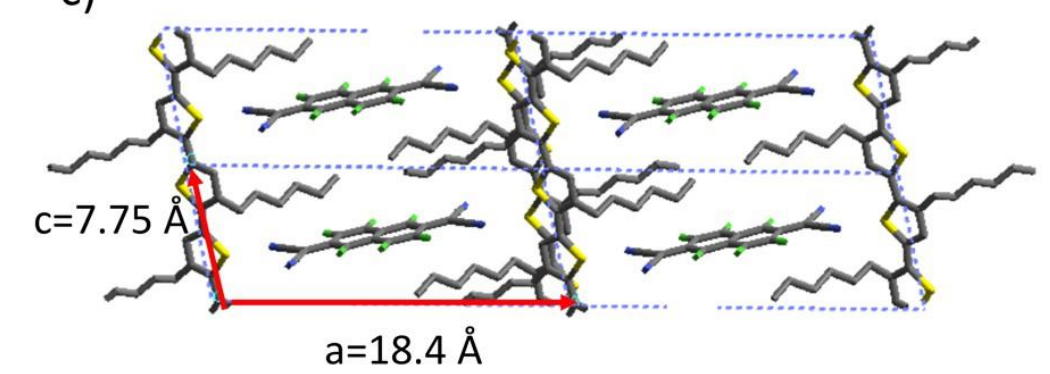

Figure 6. b-axis projections of the structural models of a) P3HT (form I), ii) the trial structure of P3HT-F ${ }_{6} T C N N Q$ and c) the final refined structure after energy minimization. Hydrogen atoms are omitted for clarity.

As seen in Figure 6.b, it is possible to create cavities by translating every second PT backbone along the $\mathbf{c}$ axis to regroup the side chains in planes that are roughly perpendicular to the polythiophene backbone. The size of the cavities depends on the dopant's geometry and dimensions. In the case of rigid planar dopants such as $\mathrm{F}_{4} \mathrm{TCNQ}$ and $\mathrm{F}_{6} \mathrm{TCNNQ}$, the cavity is determined by the dopant's long axis dimension. A longer dopant molecule induces a larger lattice expansion, as verified in the present case for $\mathrm{F}_{4} \mathrm{TCNQ}$ and $\mathrm{F}_{6} \mathrm{TCNNQ}$. This is consistent with most experimental observations showing that doping induces an extension of the lattice along the a axis (alkyl side chain direction). [26,27] 
The trial structure (Figure 6b) is an orthorhombic unit cell that is doubled along the a-axis direction (side chains) with parameters $a=39.0 \AA, b=7.8 \AA, c=7.75 \AA$. It contains two dopants at positions $(0.250 .50 .25)$ and $(0.75,0.50 .75)$. The $C_{2 / c}$ space group is suggested from the extinction rules:

$$
\begin{aligned}
& \text { h } 01 \text { with } h+l=2 n \\
& \text { h } 00 \text { with } h=2 n \text { and } \\
& 0 \text { k } 0 \text { with } k=2 n
\end{aligned}
$$

The calculated ED pattern for this trial structure reproduces very well the additional observed reflections indexed as 101 and 103 and the intense 002 reflection.

In a second step, we subjected this trial structure to energy minimization using only van der Waals interactions. The energy-minimized unit cell is triclinic with P1 space group. It is defined by $a=38.44 \AA, b=8.96 \AA, c=7.75 \AA, \alpha=107.96^{\circ}, \beta=85.5^{\circ}$ and $\gamma=106.25^{\circ}$. This "double" cell can be further reduced into a single unit cell with P-1 symmetry with a=18.8 $\mathrm{b}=8.95 \AA, c=7.75 \AA, \alpha=107.6^{\circ}, \beta=101.5^{\circ}$ and $\gamma=89.3^{\circ}$. The corresponding calculated ED pattern in Figure 5a accounts quite well for the presence of the additional reflections and for the enhanced intensity of the 002 reflection. The additional reflections in the $\ell=1$ layer line are indexed as h 01 with $h=-2,-1,0$ and 1 . This model yields an interlayer spacing $d_{100}=18.4$ $\AA$ and a $\pi$-stacking periodicity $\mathrm{d}_{\pi}=3.45 \AA$ in close concordance with the experimental values (see Table 1). The asymmetry in the intensity of the h 01 reflections is related to the triclinic character of the unit cell.

Let us describe more precisely the refined structural model. Within $\pi$-stacks, the intercalation of dopants results in i) the increase of the distance between PT backbones along the $\mathbf{b}$ axis and ii) an increase of the backbone tilt with respect to the $\mathbf{b}$ axis. PT backbones are contained in 320 planes with a short $3.46 \AA$ reticular distance corresponding to the apparent $\pi$-stacking distance $d_{\pi}$. The 2 -2 0 planes containing side chains should also result in a strong reflection. However, this reflection is not observed experimentally because in the real doped structure, 
hexyl side chains are quite disordered contrary to the ideal calculated model. This disorder possibly accounts also for the larger $\mathrm{d}_{100}$ due to the non-optimal packing of alkyl side chains.

It is highly instructive to compare the refined structure of $\mathrm{F}_{6}$ TCNNQ-doped P3HT with the structure of other doped polythiophenes. Tashiro et al. investigated the structure of P3HT doped with iodine. [33] They established that iodine doped P3HT possesses a "tunnel" structure due to the incorporation of polyiodide ions such as $\mathrm{I}_{3}{ }^{-}$and $\mathrm{I}_{5}{ }^{-}$into the layers of the alkyl side chains. Structural modeling indicated that the polyiodide chains are oriented along the $\mathbf{b}$ axis of P3HT. By contrast, in the present case, the long axis of $\mathrm{F}_{6}$ TCNNQ is oriented along the a axis (side chain direction). Most interestingly, as in our case, the introduction of dopants in the unit cell causes a shift of PT chains along the c-axis to generate the "cavities" along the $\mathbf{b}$-axis wherein dopant anions can be hosted.

The refined structure of P3HT-F TCNNQ bears also similarity with that of PEDOT doped with Tosylate (Tos). The structure of PEDOT:Tos was first analyzed by Aasmunsveit et al. using X-ray diffraction and was further modeled by Kim and Brédas. [34,35] Both doped polymers adopt a lamellar structure with layers of highly $\pi$-stacked poly(thiophene) backbones separated by layers of dopants. Moreover, the stoichiometry of the doped phases of P3HT:F 6 TCNNQ and of PEDOT:Tos is one dopant per four thiophene monomers i.e. a maximum doping concentration of approximately $25 \%$. For $\mathrm{F}_{4} \mathrm{TCNQ}$, this stoichiometry is about twice the apparent doping level extracted by UV-vis-NIR absorption spectroscopy (see table 2). This is not surprising since the P3HT films oriented by high-T rubbing at $186^{\circ} \mathrm{C}$ have a crystallinity of 50-60\% and the amorphous phase is poorly doped (no polaron seen for light polarization perpendicular to the chain direction). [27]

In a broader perspective, the growth of intercalated structures upon doping of P3HT with dopant molecules is also in line with the observation of bimolecular crystal formation between PBTTT and phenyl- $\mathrm{C}_{71}$-butyric acid methyl ester $\left(\mathrm{PC}_{71} \mathrm{BM}\right)$. [36-38] It has been shown that $\mathrm{PC}_{71} \mathrm{BM}$ molecules intercalate in the structure of PBTTT and form bimolecular 
crystals of $\mathrm{PC}_{71} \mathrm{BM}$ and $\mathrm{C}_{14}$-PBTTT. As in the present case, the bimolecular crystal phase is expanded along the alkyl side chain direction and the $\pi$-stacking characteristics are modified.

Table 1. Main structural characteristics of the doped P3HT crystal structures refined from electron diffraction and following energy minimization.

\begin{tabular}{|c|c|c|}
\hline Dopant & $\mathrm{F}_{6}$ TCNNQ & $\mathrm{F}_{4}$ TCNQ \\
\hline $\mathrm{d}_{\pi}(\AA)$ experimental & $3.55 \pm 0.05$ & $3.55 \pm 0.05$ \\
\hline $\mathrm{d}_{\pi}(\AA)$ calculated & 3.42 & 3.46 \\
\hline $\mathrm{d}_{100}(\AA ̊)$ experimental & 19.2 & 18.0 \\
\hline $\mathrm{d}_{100}(\AA)$ calculated & 18.4 & 1.36 \\
\hline Density $\left(\mathrm{g} / \mathrm{cm}^{3}\right)$ & 1.40 & -96.6 \\
\hline $\mathrm{E}\left(\mathrm{kCal} / \mathrm{mol}^{3}\right)$ & -106.9 & \\
\hline
\end{tabular}

To consolidate this result, we used the same approach for the structure of P3HT: $\mathrm{F}_{4} \mathrm{TCNQ}$ (see Figure ESI5). We also find a stable intercalated structure (see Table 1). The $d_{\pi}$ and $d_{100}$ periodicities vary upon doping with $\mathrm{F}_{4} \mathrm{TCNQ}$ as for $\mathrm{F}_{6} \mathrm{TCNNQ}$ : reduction of $\pi$-stacking distance and expansion along the alkyl side chain direction. However, there is one major difference between $\mathrm{F}_{4}$ TCNQ and $\mathrm{F}_{6}$ TCNNQ-doped structures. No additional h 01 reflections are seen as in the case of $\mathrm{F}_{6} \mathrm{TCNNQ}$. One can only evidence the enhanced intensity of the 00 2 and the changes in lattice dimensions for $\mathrm{F}_{4}$ TCNQ-doped P3HT. A difference in the ordering of $\mathrm{F}_{4} \mathrm{TCNQ}$ and $\mathrm{F}_{6} \mathrm{TCNNQ}$ in the P3HT crystal is a possible reason for this. Indeed, polarized UV-vis-NIR spectroscopy evidences a better ordering of $\mathrm{F}_{6} \mathrm{TCNNQ}$ as compared to $\mathrm{F}_{4} \mathrm{TCNQ}$. Following the recent work by Vijayakumar et al., $\mathrm{F}_{4} \mathrm{TCNQ}$ diffuses more readily into the $\mathrm{C}_{12}$-PBTTT film than $\mathrm{F}_{6}$ TCNNQ. [21] As a consequence, $\mathrm{F}_{6}$ TCNNQ is better ordered in the $\mathrm{C}_{12}$-PBTTT lattice than $\mathrm{F}_{4}$ TCNQ due to its slower diffusion. Moreover, the diffusion of $\mathrm{F}_{4} \mathrm{TCNQ}$ is significantly faster in P3HT than in $\mathrm{C}_{12}$-PBTTT (diffusion times into 100nm-thick films are of the order of a few seconds for P3HT and 40-50 s for $\mathrm{C}_{12}$-PBTTT). 
Accordingly, both ED and spectroscopy results clearly suggest larger disorder in the intercalated $\mathrm{F}_{4} \mathrm{TCNQ}$ than $\mathrm{F}_{6} \mathrm{TCNNQ}$.

More generally, for both dopants, the structure found in the films is much more disordered than the ideal model arrived at by the ED analysis. In particular, streaking in the ED pattern along the $\mathbf{a}$ axis is clear evidence for disorder in the arrangement of successive $(b, c)$ planes of stacked PTs.

To conclude, electron diffraction provides compelling evidence for the presence of dopant molecules inside the unit cell of P3HT. Dopants are intercalated in the layers of alkyl side chains in a plane perpendicular to the polythiophene chains. Rearrangement of P3HT chains generates two cavities within a doubled unit cell that can host two dopant molecules. One question remains: why the additional reflections in the $\ell=1$ layer are not observed in the case of $\mathrm{F}_{4} \mathrm{TCNQ}$ ? To answer this question, we used polarized UV-vis-NIR spectroscopy and more precisely angular dependent spectroscopy to try to quantify the ordering of dopant molecules inside the P3HT host matrix.

\subsection{Probing the ordering of $F_{4}$ TCNQ and $F_{6}$ TCNNQ dopants in the polymer matrix of P3HT using polarized UV-vis-NIR spectroscopy.}

Polarized UV-vis-NIR spectroscopy of aligned P3HT was used to clarify the orientation distribution of dopant molecules in the polymer matrix. To this aim, UV-vis-NIR spectra were recorded as a function of the angle between the P3HT chain direction (rubbing direction) and the direction of the light polarization. This helped quantify the relative order parameters of polymer chains and radical anions in the oriented thin films. Figure 7.a,b show the two sets of polarized UV-vis-NIR spectra recorded every $10^{\circ}$ within the $0^{\circ}-90^{\circ}$ range. To extract the angular dependence of the dopant's absorption, we used a multipeak analysis to remove the underlying polaronic contributions of the polymer (see experimental section and Figure ESI 6). The two graphs in Figure 7.c and 7.d illustrate the angular dependences of 
the polaron ( $\mathrm{P} 1$ peak at $2500 \mathrm{~nm}$ ) and of the $\mathrm{F}_{4} \mathrm{TCNQ}^{-}$and $\mathrm{F}_{6} \mathrm{TCNNQ}^{-}$radical anions, respectively. The radical anion absorptions at $873 \mathrm{~nm}\left(\mathrm{~F}_{4} \mathrm{TCNQ}^{-}\right)$and $1138 \mathrm{~nm}\left(\mathrm{~F}_{6} \mathrm{TCNNQ}^{-}\right)$ for each angular position are obtained by subtracting the polaronic contribution of P3HT.

a)

Figure 7: a) ai

films doped wi

with respect $t$

parallel to the

light polarized c)

dependence of

dopant anion ,

$F_{6} T C N N Q^{-}(d)$.

text of the SI.

e)

the polymer an
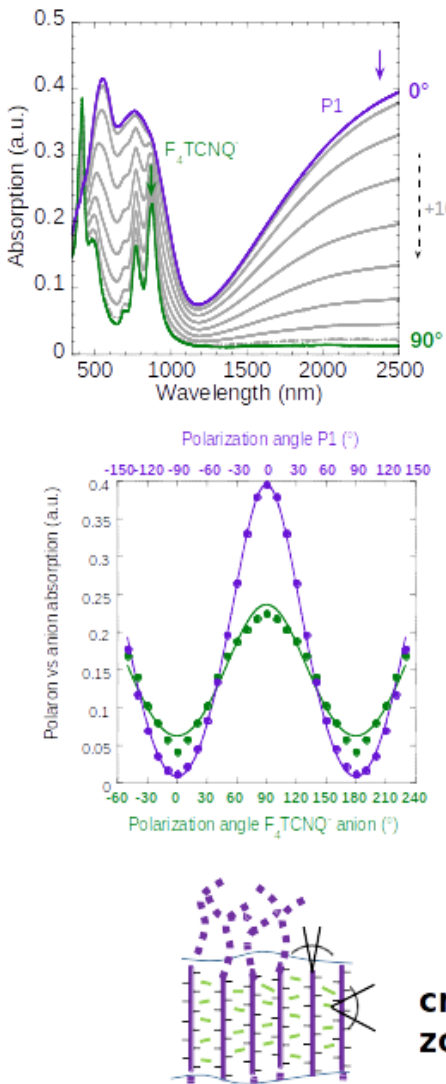

Comparison of
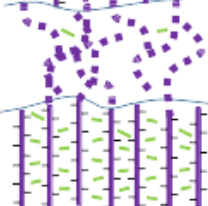

the in-plane b)

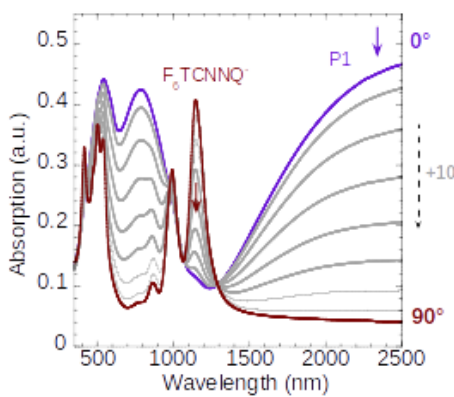

lucting P3HT

gle measured

ght polarized

o the incident

d) Polarization angle P1 $\left({ }^{\circ}\right)$

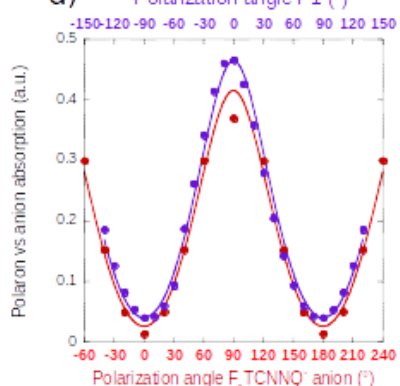

), d) Angular

2), and of the

red) for the

$\imath$ given in the

ment between

f)

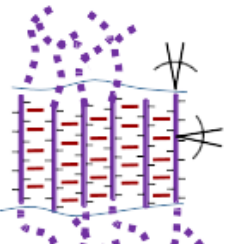

crystalline

zones

amorphous

regions

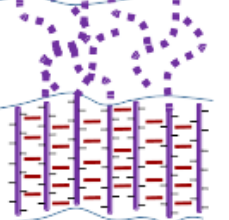

cal anions of

a measure of

dopants gives an idea of the way the dopants are oriented with respect to the polymer crystals when intercalated in the layers of alkyl side chains. Similar OPs for anion and polaron bands suggest that the orientation of the dopant follows strictly the orientation of the side chains perpendicular to the PT backbones. The values of the OP in Table 2 show an obvious trend: for $\mathrm{F}_{6} \mathrm{TCNNQ}$, the OPs of polaron and radical anion are almost identical whereas they are substantially different for the $\mathrm{F}_{4} \mathrm{TCNQ}^{-}$radical anions. This suggests that $\mathrm{F}_{4} \mathrm{TCNQ}$ anions are oriented in a more random manner in the P3HT lattice (see schematic illustration of the 
$\mathrm{F}_{4} \mathrm{TCNQ}$ and $\mathrm{F}_{6} \mathrm{TCNNQ}$ molecules orientation with respect to the polymer backbone in Figure 7e and 7f). Accordingly, the intercalation of $\mathrm{F}_{6}$ TCNNQ seems to lead to a more ordered structure of the doped phase of P3HT than for $\mathrm{F}_{4} \mathrm{TCNQ}$. This is consistent with the results of TEM showing well-defined additional reflections, accounting for a regular lattice of $\mathrm{P} 3 \mathrm{HT} / \mathrm{F}_{6} \mathrm{TCNNQ}$. The absence of $h 01$ reflections for $\mathrm{F}_{4} \mathrm{TCNQ}$ is therefore a fingerprint for a more disordered intercalation of the dopants in the unit cell of P3HT, consistent with the results obtained from polarized UV-vis-NIR spectroscopy. In the case of $\mathrm{F}_{4} \mathrm{TCNQ}$, the angular distribution is larger, whereas in case of $\mathrm{F}_{6} \mathrm{TCNNQ}$ the degree of orientation is high as evidenced by similar OP of polymer and the dopant (see Table 2). It is worth to note that a similar effect has been observed for doped $\mathrm{C}_{12}$-PBTTT films. [23] The lower ordering of $\mathrm{F}_{4} \mathrm{TCNQ}$ was explained by the fact that the diffusion coefficient of this dopant in the polymer film is almost one order of magnitude larger as compared to $\mathrm{F}_{6} \mathrm{TCNNQ}$. A faster diffusion of $\mathrm{F}_{4} \mathrm{TCNQ}$ could accordingly explain the more disordered distribution of the dopant orientation in the side chain layers of P3HT. However, contrary to $\mathrm{C}_{12}$-PBTTT, the diffusion kinetics of doping could not be followed by UV-vis-NIR spectroscopy as it is even faster for P3HT than for $\mathrm{C}_{12}$-PBTTT.

Table 2: Main characteristics of oriented P3HT films $\left(T_{R}=180^{\circ} \mathrm{C}\right)$ sequentially doped with $F_{4} T C N Q^{-}$and $F_{6} T C N N Q^{-}$.

\begin{tabular}{|c|c|c|}
\hline Main characteristics of doped phases & P3HT: $F_{4}$ TCNQ & P3HT: $F_{6}$ TCNNQ \\
\hline Order parameter polaron & $0.91 \pm 0.05$ & $0.76 \pm 0.05$ \\
\hline Order parameter anion & $0.62 \pm 0.05$ & $0.82 \pm 0.05$ \\
\hline $\mathrm{d}_{020}(\AA)$ & $3.61 \pm 0.05$ & $3.63 \pm 0.05$ \\
\hline $\mathrm{d}_{100}(\AA)$ & $18.1 \pm 0.1$ & $19.2 \pm 0.1$ \\
\hline Anion per 100 thiophene (\%) & $14 \pm 2$ & $20 \pm 2$ \\
\hline
\end{tabular}




\begin{tabular}{|c|c|c|}
\hline Charge conductivity $\sigma_{/ /}\left(\mathrm{S} \mathrm{cm}^{-1}\right)$ & 287 & 521 \\
\hline Power factor $\mathrm{PF} / / \max \left(\mu \mathrm{W} \mathrm{m}{ }^{-1} \mathrm{~K}^{-2}\right)$ & $73 \pm 5$ & $79 \pm 30$ \\
\hline
\end{tabular}

\subsection{Resulting thermoelectrical properties of doped films.}

Finally, we evaluate the impact of the structural evolution with doping concentration on the charge conductivity and thermopower. Figure 8 features the evolution of the charge conductivity, Seebeck coefficient and power factor as a function of dopant concentration for aligned P3HT films doped with $\mathrm{F}_{4} \mathrm{TCNQ}$ and $\mathrm{F}_{6} \mathrm{TCNNQ}$. In case of $\mathrm{F}_{4} \mathrm{TCNQ}$, the conductivity is already saturated at $1 \mathrm{~g} / \mathrm{l}$ reaching the value of $287 \mathrm{~S} / \mathrm{cm}$, whereas for $\mathrm{F}_{6}$ TCNNQ the conductivity is almost saturated at $10 \mathrm{~g} / \mathrm{l}$ at $521 \mathrm{~S} / \mathrm{cm}$. The larger conductivity for $\mathrm{F}_{6} \mathrm{TCNNQ}$ is consistent with the slightly larger oxidation level evidenced by UV-vis-NIR spectroscopy (see Figure 2b) and the larger dopant concentration extracted from UV-vis-NIR absorption spectroscopy. The same trend is observed in the perpendicular direction, reaching its maximum at $56 \mathrm{~S} / \mathrm{cm}$ and $107 \mathrm{~S} / \mathrm{cm}$ for $\mathrm{F}_{4} \mathrm{TCNQ}$ and $\mathrm{F}_{6} \mathrm{TCNNQ}$, respectively. It is worth to note that the conductivity reached here for $\mathrm{F}_{4} \mathrm{TCNQ}$ is larger than that reported in reference $27(150 \mathrm{~S} / \mathrm{cm})$. This difference is due to the fact that incremental concentration doping was used in this study (see Figure $1 \mathrm{c}$ ). ICD of oriented $\mathrm{C}_{12}$-PBTTT films doped with $\mathrm{F}_{4} \mathrm{TCNQ}$ and $\mathrm{F}_{6} \mathrm{TCNNQ}$ leads to a substantial increase of charge conductivities. [23] The improved conductivity is related to the fact that ICD preserves best the pristine structure of the films and hence helps reach larger charge conductivities.

Regarding the Seebeck coefficient, it tends to decrease with increasing dopant concentration. This dependence looks pretty similar for both dopant molecules, with values reaching 45 $\mu \mathrm{V} / \mathrm{K}$ and $15 \mu \mathrm{V} / \mathrm{K}$ parallel and perpendicular to the rubbing direction, respectively. The anisotropy of conductivity is always larger than that of the Seebeck coefficient which is consistent with previous studies $[26,27,30]$ 
Regarding the power factor PF for both systems, similar trends are observed for both dopants. The larger conductivity for P3HT films doped with $\mathrm{F}_{6}$ TCNNQ is not sufficient to counter-balance the reduction of the Seebeck factor. The PF values saturate at around 100 $\mu \mathrm{W} \mathrm{m} \mathrm{m}^{-1} \mathrm{~K}^{-2}$ and $1 \mu \mathrm{W} \mathrm{m} \mathrm{m}^{-1} \mathrm{~K}^{-2}$ in parallel and perpendicular directions to the rubbing, respectively. The highest PF obtained in this study is slightly below the recent record value of $160 \mu \mathrm{W} \mathrm{m}{ }^{-1} \mathrm{~K}^{-2}$ in P3HT thin films doped with the Molybdenum complex Mo(tfd-COCF 3$)_{3}$. [30] Accordingly, while improved dopant ordering is likely the reason for the higher charge conductivity for $\mathrm{F}_{6} \mathrm{TCNNQ}$, it is not sufficient to improve TE properties in thin films as the Seebeck coefficient is, expectedly, reduced. The comparison of the results between $\mathrm{F}_{4} \mathrm{TCNQ}$, $\mathrm{F}_{6}$ TCNNQ and $\mathrm{Mo}\left(\mathrm{tfd}-\mathrm{COCF}_{3}\right)_{3}$ suggests that the chemical nature of the dopant is essential in maximizing the TE PF. This is possibly related to the shielding of Coulombic interactions between polaron and counterions that determines the polaron delocalization, hence charge mobility. In this regard $\mathrm{Mo}\left(\mathrm{tfd}-\mathrm{COCF}_{3}\right)_{3}$ must be particularly effective as compared to $\mathrm{F}_{6}$ TCNNQ. Moreover, the observed difference in TE performances might also relate to the difference in doping of crystalline and amorphous domains observed for Motfd$\left.\mathrm{COCF}_{3}\right)_{3 \cdot} \cdot[29,30]$

Figure 8: Evolution of the a) cdoargecedAductivity $\mathrm{R}$ ) . Seebeck Coefficient, and c) power factor in oriented semi-crysta 500 ing $F_{6} T C N N Q$ (dark red). The me $\begin{array}{r}\text { ठु } 200 \\ \perp \mathbf{R} \\ \hline\end{array}$

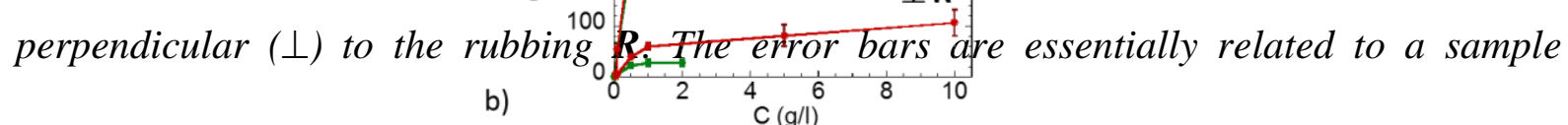
dispersion in alignment degree.

e. 160

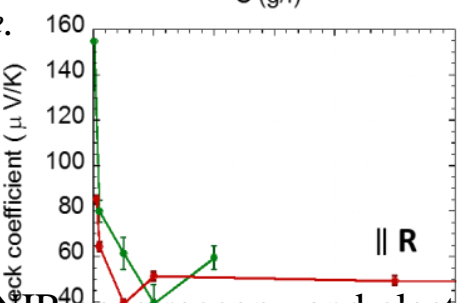

3. Conclusions.

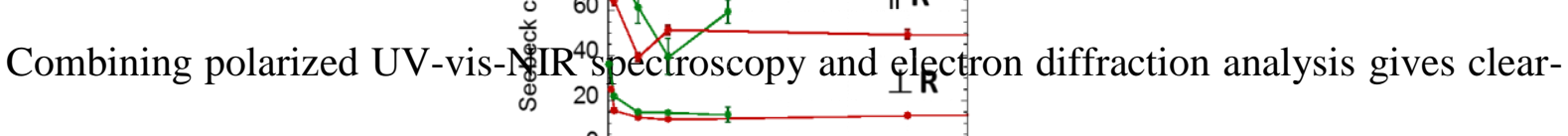

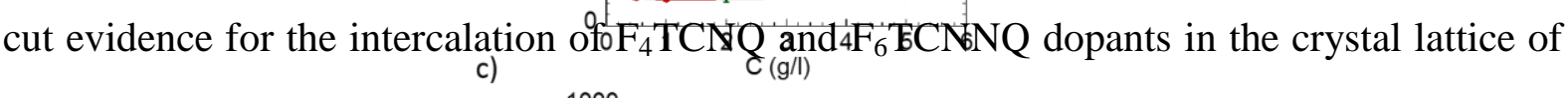

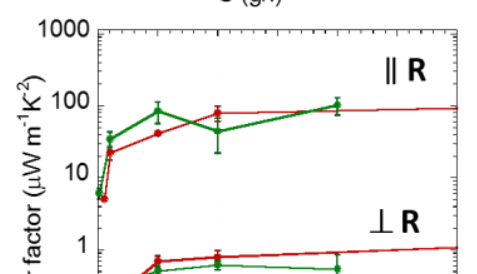


P3HT. Differences in doping mechanism have been highlighted. F $_{6}$ TCNNQ is intercalated in a more ordered manner than $\mathrm{F}_{4} \mathrm{TCNQ}$. A tentative structural model based on ED analysis is proposed for $\mathrm{P} 3 \mathrm{HT}: \mathrm{F}_{6} \mathrm{TCNNQ}$ leading to a triclinic structure with $\mathrm{P}-1$ symmetry $(\mathrm{a}=18.8 \AA$, $\mathrm{b}=8.95 \AA, c=7.75 \AA, \alpha=107.6^{\circ}, \beta=101.5^{\circ}$ and $\gamma=89.3^{\circ}$ ). The structure of doped P3HT bears similarity with the structure of PEDOT:Tos in terms of P3HT/dopant stoichiometry. Despite larger charge conductivities for $\mathrm{F}_{6} \mathrm{TCNNQ}$, the power factors remain similar for both dopants with a maximum in the range $70-80 \mu \mathrm{W} \mathrm{m} \mathrm{m}^{-1}$. This study demonstrates that highly ordered P3HT/dopant intercalates can be prepared by sequential doping. The level of ordering of dopants in the polymer crystals might be further optimizing the kinetics of dopant diffusion or the geometry of the dopants. Adapting the dopant dimensions to the possible cavities in the layers of side-chains in the P3HT lattice might be an interesting strategy to design highly ordered P3HT:dopant intercalates with enhanced optoelectronic properties.

Acknowledgements: We thank the Agence Nationale de la Recherche for financial support through project ANR -17-CE05-0012 (ANISOTHERM) and CNRS through PEPS grant Thermobody. M. Schmutz and C. Blanck are acknowledged for technical support in TEM. Funding from Region Grand'Est is acknowledged for P. Durand.

\section{Experimental section}

Orientation and doping of thin films: regio-regular P3HT was purchased from Merck $\left(\mathrm{M}_{\mathrm{w}}=43.6 \mathrm{kDa}, \mathrm{PDI}=1.8\right) . \mathrm{F}_{4} \mathrm{TCNQ}$ powder and anhydrous solvents such as acetonitrile and ortho-dichlorobenzene (oDCB) were obtained from Sigma-Aldrich and used without purification. $\mathrm{F}_{6}$ TCNNQ was synthesized following the procedure given in reference 21 . The films were prepared by doctor-blading a hot solution of P3HT in $o \mathrm{DCB}\left(10 \mathrm{mg} \mathrm{mL}^{-1}\right)$ at $160^{\circ} \mathrm{C}$ on glass slide substrates pre-covered with a sacrificial hydrosoluble NaPSS (10 mg $\mathrm{mL}^{-1} \mathrm{aq)}$ layer. The orientation of the films by high- $\mathrm{T}$ rubbing followed the protocol described in previous publications. [26,27,31] Rubbing is performed by using a homemade 
set-up consisting of a rotating cylinder covered with a microfiber cloth and a translating hot plate. The film thickness was extracted from the UV-vis absorbance using the calibration given in reference 30 .

The doping was performed following the incremental concentration doping (ICD) procedure [23] with full sample immersion for $10 \mathrm{~s}$ in the dopant solution of increasing concentration. Such short doping times are sufficient to reach saturation of the film doping level as shown earlier by Jacobs et al. [10] No rinsing step was conducted as it can lead to dedoping of the films. Both doping and rubbing were performed under nitrogen atmosphere.

Structural analysis by TEM. The polymer films were coated with a thin amorphous carbon film and removed from the glass substrate by floating on water and subsequent recovery on TEM copper grids. TEM was performed in bright field and diffraction modes using a CM12 Philips microscope $(120 \mathrm{kV})$ equipped with a MVIII (Soft Imaging System) charge coupled device camera.

Calibration of the reticular distances in the ED patterns was made using the 002 monomer periodicity reflection at $3.85 \AA$ that is not sensitive to the doping of the polymer. Beam exposure was set to a minimum using the low dose system to avoid de-doping under the electron beam that is observed when the same zone is exposed for a prolonged period of time. De-doping is clearly manifested in the ED pattern by a change in reticular distances to those of the pristine undoped P3HT films.

Polarized UV-Vis-NIR absorption. The orientation of the polymer films was probed by UV-vis-NIR absorption (350-2500 nm) using a Varian Cary5000 spectrometer with polarized incident light (spectral resolution of $1 \mathrm{~nm}$ ).

In order to deduce the pure anionic signal, the UV-vis-NIR spectra were recorded every $10^{\circ} / 20^{\circ}$. The angle of light polarization is measured with respect to the rubbing direction. A multiple peak fitting was applied in order to deconvolve the recorded spectra using the PeakFit program. The results of this analysis are presented in the Supporting information. 
The whole set of spectra in both parallel and in perpendicular to the rubbing were measured as a function of the doping concentration, on the Figure $2 \mathrm{~d}$ the normalized doping level calculated using the ratio $C_{\text {thiophene }} / C_{\text {dopant anion }}, C_{\text {thiophene }}$ and $C_{\text {dopant anion }}$ are the molar concentrations of thiophenes and dopant anions, respectively. The nominator can be easily calculated from the known unit cell parameters and from the fact that we have 4 thiophene rings in the unit cell, so the thiophenes concentration can be found as $\frac{4}{V}$, where $\mathrm{V}$ is the volume of the doped unit cell. The dopant anion concentration was calculated from the Beer-Lambert-Bouguer law $A=\varepsilon \cdot l \cdot C$, where $\mathrm{A}$ is the absorbance of the attenuating species, $\varepsilon$ - the extinction coefficient of the anion (50000 $1 \mathrm{Mol}^{-1} \mathrm{~cm}^{-1}$ for $\mathrm{F}_{4}$ TCNQ and $53700 \mathrm{l}^{1} \mathrm{Mol}^{-1} \mathrm{~cm}^{-1}$ for $\left.\mathrm{F}_{6} \mathrm{TCNNQ}[23]\right), l-$ the film thickness and $C$ - the molar concentration of the anion.

Charge conductivity and Seebeck coefficient. All devices were fabricated on glass substrates cleaned by ultrasonication in acetone, ethanol, hellmanex and deionized water (x3 times). The cleaned substrates were dried under nitrogen and exposed to plasma prior to film deposition. Gold electrical contacts (40 nm thick) in a four-points probe geometry $(1 \mathrm{~mm}$ spacing between electrodes, $5 \mathrm{~mm}$ length) were deposited via controlled thermal evaporation through a shadow mask, at an average rate of $4-6 \AA /$ s. The geometry of deposited gold electrodes allows determining the charge transport and thermopower on a same substrate in both parallel and perpendicular directions to rubbing. Oriented films of P3HT were floated on water and carefully recovered on the device with pre-deposited gold electrodes. They were subsequently doped using the ICD protocol. [23]

Four-point probe measurements of electrical conductivity were performed using a Keithley 4200-SCS and a Lab Assistant Semiprobe station in a Jacomex glovebox under $\mathrm{N}_{2}$ atmosphere. The resistivity $\rho$ was derived from the sheet resistance $R$ such that $\rho=1.81 \cdot \mathrm{R} t$ 
where $\mathrm{t}$ is the film thickness (the geometrical correction factor was determined following the method in reference 31 ). The average conductivity value for a given rubbing temperature was taken as the average of two to four devices.

Thermopower measurements were conducted in nitrogen atmosphere on the same devices. The thermopower was measured using a differential temperature method whereby a temperature gradient was established across the sample along or perpendicular to the rubbing direction. Calibration of the Seebeck coefficient measurement was performed using a constantan wire. The detailed procedure is given in references 23 and 31 .

\section{Conflict of interest.}

The authors declare no conflicts of interest.

\section{Supporting Information.}

Comparison of the electron diffraction patterns for films doped using the ICD and direct doping methods; Concentration-dependence of the polarized UV-vis-NIR spectra for P3HT films doped with $\mathrm{F}_{4} \mathrm{TCNQ}$ and $\mathrm{F}_{6} \mathrm{TCNNQ}$; Correlation between the P1 band absorbance and the anion absorbance; Plot profile of the ED patterns for doped P3HT with $\mathrm{F}_{6} \mathrm{TCNNQ}(5 \mathrm{~g} / \mathrm{l})$

for $1=0,1$; Angular dependence of the anion absorption peak after removal of polaronic contributions; Concentration-dependence of the anisotropies of the Seebeck coefficient and charge conductivity. 


\section{References}

[1] Ogawa, S. in Organic Electronics, Springer Japan, 2015, pp 1-245.

[2] Goel, M.; Thelakkat, M. Polymer Thermoelectrics: Opportunities and Challenges. Macromolecules 2020, 53 (10), 3632-3642.

[3] Vijayakumar, V.; Zaborova, E.; Biniek, L.; Zeng, H.; Herrmann, L.; Carvalho, A.; Boyron, O.; Leclerc, N.; Brinkmann, M. Impact of Alkyl Side Chain Length on Doping Kinetics, Thermopower and Charge Transport Properties in Highly Oriented F4TCNQ-Doped PBTTT Films. ACS Appl. Mater. Interfaces 2019, 11, 4942.

[4] Kroon, R.; Kiefer, D.; Stegerer, D.; Yu, L.; Sommer, M.; Müller, C. Polar Side Chains Enhance Processability, Electrical Conductivity, and Thermal Stability of a Molecularly pDoped Polythiophene. Advanced Materials 2017, 29 (24), 1700930.

[5] Jacobs, I. E.; Moulé, A. J. Controlling Molecular Doping in Organic Semiconductors. Advanced Materials 2017, 29 (42), 1703063.

[6] Bubnova, O. and Crispin, X. Towards polymer-based organic thermoelectric generators. Energy Environ. Sci. 2012, 5, 9345.

[7] Cochran, J. E.; Junk, M. J. N.; Glaudell, A. M.; Miller, P. L.; Cowart, J. S.; Toney, M. F.; Hawker, C. J.; Chmelka, B. F.; Chabinyc, M. L. Molecular Interactions and Ordering in Electrically Doped Polymers: Blends of PBTTT and F4TCNQ. Macromolecules 2014, 47 (19), 6836-6846.

[8] Patel, S. N.; Chabinyc, M. L. Anisotropies and the Thermoelectric Properties of Semiconducting Polymers. Journal of Applied Polymer Science 2017, 134 (3).

[9] Patel, S. N.; Glaudell, A. M.; Peterson, K. A.; Thomas, E. M.; O’Hara, K. A.; Lim, E.; Chabinyc, M. L. Morphology Controls the Thermoelectric Power Factor of a Doped Semiconducting Polymer. Science Advances 2017, 3 (6).

[10] Jacobs, I. E.; Aasen, E. W.; Oliveira, J. L.; Fonseca, T. N.; Roehling, J. D.; Li, J.; Zhang, G.; Augustine, M. P.; Mascal, M.; Moulé, A. J. Comparison of Solution-Mixed and 
Sequentially Processed P3HT:F4TCNQ Films: Effect of Doping-Induced Aggregation on Film Morphology. J. Mater. Chem. C 2016, 4 (16), 3454-3466.

[11] Hynynen, J.; Kiefer, D.; Müller, C. Influence of Crystallinity on the Thermoelectric Power Factor of P3HT Vapour-Doped with F4TCNQ. RSC Adv. 2018, 8 (3), 1593-1599.

[12] Scholes D. Tyler; Yee Patrick Y.; Lindemuth Jeffrey R.; Kang Hyeyeon; Onorato Jonathan; Ghosh Raja; Luscombe Christine K.; Spano Frank C.; Tolbert Sarah H.; Schwartz Benjamin J. The Effects of Crystallinity on Charge Transport and the Structure of Sequentially Processed F4TCNQ-Doped Conjugated Polymer Films. Advanced Functional Materials 2017, 27 (44), 1702654.

[13] Scholes, D. T.; Hawks, S. A.; Yee, P. Y.; Wu, H.; Lindemuth, J. R., Tolbert, S. H.; Schwartz, B. J. Overcoming Film Quality Issues for Conjugated Polymers Doped with F4TCNQ by Solution Sequential Processing: Hall Effect, Structural, and Optical Measurements. J. Phys. Chem. Lett. 2015, 6, 4786.

[14] Scholes, D. T.; Yee, P. Y.; Lindemuth, J. R.; Kang, H.; Onorato, J.; Ghosh, R.; Luscombe, C. K.; Spano, F. C.; Tolbert, S. H.; Schwartz, B. J. The Effects of Crystallinity on Charge Transport and the Structure of Sequentially Processed F4TCNQ-Doped Conjugated Polymer Films. Advanced Functional Materials 2017, 27 (44), 1702654.

[15] Kroon, R.; Mengistie, D. A.; Kiefer, D.; Hynynen, J.; Ryan, J. D.; Yu, L.; Müller, C. Thermoelectric Plastics: From Design to Synthesis, Processing and Structure-Property Relationships. Chem. Soc. Rev. 2016, 45 (22), 6147-6164.

[16] Glaudell, A. M.; Cochran, J. E.; Patel, S. N.; Chabinyc, M. L. Impact of the Doping Method on Conductivity and Thermopower in Semiconducting Polythiophenes. Advanced Energy Materials 2015, 5 (4), 1401072.

[17] Pingel, P.; Neher, D. Comprehensive Picture of p-Type Doping of P3HT with the Molecular Acceptor F4TCNQ. Phys. Rev. B 2013, 87 (11), 115209.

[18] Karpov, Y.; Erdmann, T.; Stamm, M.; Lappan, U.; Guskova, O.; Malanin, M.; Raguzin, I.; Beryozkina, T.; Bakulev, V.; Günther, F.; Gemming, S.; Seifert, G.; Hambsch, M.; 
Mannsfeld, S.; Voit, B.; Kiriy, A. Molecular Doping of a High Mobility Diketopyrrolopyrrole-Dithienylthieno[3,2-b]Thiophene Donor-Acceptor Copolymer with F6TCNNQ. Macromolecules 2017, 50 (3), 914-926.

[19] Tietze, M., Burtone, L., Riede, M., Lüssem, B. \& Leo, K. Fermi Level Shift and Doping Efficiency in p-Doped Small Molecule Organic Semiconductors: A Photoelectron Spectroscopy and Theoretical Study. Phys. Rev. B 2012, 86, 035320.

[20] Lim, E.; Peterson, K. A.; Su, G. M.; Chabinyc, M. L. Thermoelectric Properties of Poly(3-Hexylthiophene) (P3HT) Doped with 2,3,5,6-Tetrafluoro-7,7,8,8Tetracyanoquinodimethane (F4TCNQ) by Vapor-Phase Infiltration. Chem. Mater. 2018, 30 (3), 998-1010.

[21] Neusser, D.; Malacrida, C.; Kern, M.; Gross, Y. M.; van Slageren, J.; Ludwigs, S. High Conductivities of Disordered P3HT Films by an Electrochemical Doping Strategy. Chem. Mater. 2020, 32 (14), 6003-6013.

[22] Bruchlos, K.; Trefz, D.; Hamidi-Sakr, A.; Brinkmann, M.; Heinze, J.; Ruff, A.; Ludwigs, S. Poly(3-Hexylthiophene) Revisited - Influence of Film Deposition on the Electrochemical Behaviour and Energy Levels. Electrochimica Acta 2018, 269, 299-311.

[23] Vijayakumar, V.; Durand, P.; Zeng, H.; Untilova, V.; Herrmann, L.; Algayer, P.; Leclerc, N. and Brinkmann, M. Influence of dopant size and doping method on the structure and thermoelectric properties of PBTTT films doped with $\mathrm{F}_{6}$ TCNNQ and $\mathrm{F}_{4} \mathrm{TCNQ}$. J. Mater. Chem. C 2020, 8, 16470.

[24] Duong, D. T.; Wang, C.; Antono, E.; Toney, M. F.; Salleo, A. The Chemical and Structural Origin of Efficient P-Type Doping in P3HT. Organic Electronics 2013, 14 (5), $1330-1336$.

[25] Wang, C.; Duong, D. T.; Vandewal, K.; Rivnay, J.; Salleo, A. Optical Measurement of Doping Efficiency in Poly(3-Hexylthiophene) Solutions and Thin Films. Phys. Rev. B 2015, $91(8), 085205$. 
[26] Hamidi-Sakr, A.; Biniek, L.; Bantignies, J.-L.; Maurin, D.; Herrmann, L.; Leclerc, N.; Lévêque, P.; Vijayakumar, V.; Zimmermann, N.; Brinkmann, M. A Versatile Method to Fabricate Highly In-Plane Aligned Conducting Polymer Films with Anisotropic Charge Transport and Thermoelectric Properties: The Key Role of Alkyl Side Chain Layers on the Doping Mechanism. Advanced Functional Materials 2017, 27, 1700173.

[27] Untilova, V.; Biskup, T.; Biniek, L.; Vijayakumar, V.; Brinkmann, M. Control of Chain Alignment and Crystallization Helps Enhance Charge Conductivities and Thermoelectric Power Factors in Sequentially Doped P3HT:F4TCNQ Films. Macromolecules 2020, 53, 2441.

[28] Mladenović, M.; Vukmirović, N. Electronic States at the Interface between Crystalline and Amorphous Domains in Conjugated Polymers. J. Phys. Chem. C 2015, 119 (41), 2332923333.

[29] Hynynen, J.; Järsvall, E.; Kroon, R.; Zhang, Y.; Barlow, S.; Marder, S. R.; Kemerink, M.; Lund, A.; Müller, C. Enhanced Thermoelectric Power Factor of Tensile Drawn Poly(3Hexylthiophene). ACS Macro Lett. 2019, 8 (1), 70-76.

[30] Untilova, V.; Hynynen, J.; Hofmann, A. I.; Scheunemann, D.; Zhang, Y.; Barlow, S.; Kemerink, M.; Marder, S. R.; Biniek, L.; Müller, C.; Brinkmann, M. High Thermoelectric Power Factor of Poly(3-Hexylthiophene) through In-Plane Alignment and Doping with a Molybdenum Dithiolene Complex. Macromolecules 2020, 53 (15), 6314-6321.

[31] Hamidi-Sakr, A.; Biniek, L.; Fall, S.; Brinkmann, M. Precise Control of Lamellar Thickness in Highly Oriented Regioregular Poly(3-Hexylthiophene) Thin Films Prepared by High-Temperature Rubbing: Correlations with Optical Properties and Charge Transport. Advanced Functional Materials 2016, 26 (3), 408-420.

[32] Kayunkid, N.; Uttiya, S.; Brinkmann, M. Structural Model of Regioregular Poly(3Hexylthiophene) Obtained by Electron Diffraction Analysis. Macromolecules 2010, 43 (11), 4961-4967.

[33] Tashiro, K., Kobayashi, M., Kawai, T. \& Yoshino, K. Crystal structural change in poly(3-alkyl thiophene)s induced by iodine doping as studied by an organized combination of 
X-ray diffraction, infrared/Raman spectroscopy and computer simulation techniques. Polymer 1997, 38, 2867-2879.

[34] Aasmundtveit, K. E.; Samuelsen, E. J.; Inganäs, O.; Pettersson, L. A. A.; Johansson, T.; Ferrer, S. Structural Aspects of Electrochemical Doping and Dedoping of Poly(3,4Ethylenedioxythiophene). Synthetic Metals 2000, 113 (1), 93-97.

[35] Kim, E.-G.; Brédas, J.-L. Electronic Evolution of Poly(3,4-Ethylenedioxythiophene) (PEDOT): From the Isolated Chain to the Pristine and Heavily Doped Crystals. J. Am. Chem. Soc. 2008, 130 (50), 16880-16889.

[36] Miller, N. C.; Cho, E.; Gysel, R.; Risko, C.; Coropceanu, V.; Miller, C. E.; Sweetnam, S.; Sellinger, A.; Heeney, M.; McCulloch, I.; Brédas, J. L.; Toney, M. F. and Mc Gehee, M. D. Factors Governing Intercalation of Fullerenes and Other Small Molecules Between the Side Chains of Semiconducting Polymers Used in Solar Cells. Advanced Energy Materials 2012, 2, 1208.

[37] Miller, N. C.; Cho, E.; Junk, M. J. N.; Gysel, R.; Risko, C.; Kim, D.; Sweetnam, S.; Miller, C. E. ; Richter, L. J.; Kline, R. J.; Heeney, M.; McCulloch, I.; Amassian, A.; Acevedo-Feliz, D.; Knox, C.; Hansen, M. R.; Dudenko, D.; Chmelka, B. F.; Toney, M. F.; Brédas, J.-L. and McGehee, M. D. Use of X-Ray Diffraction, Molecular Simulations, and Spectroscopy to Determine the Molecular Packing in a Polymer-Fullerene Bimolecular Crystal. Advanced Materials 2012, 24, 6071.

[38] Yang, B. - K. Wang, C.-A. Zhang, W.-Y. and Ruan, J. Successive stepwise evolution of host layer-stacking framework upon the intercalation of mobile vapor guests within side-chain layers. J. Polym. Sci. B: Polym. Phys. 2017, 55, 1448. 